\title{
Nuevos conceptos de la reanimación hídrica intravenosa
}

\section{New concepts in intravenous fluid therapy}

\author{
Ángel A. Pérez-Calatayud" *, Manuel A. Díaz-Carrillo², Eduardo D. Anica-Malagón ${ }^{3}$ y Jesús C. Briones-Garduño ${ }^{4}$

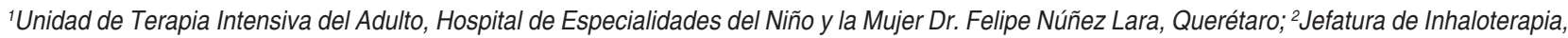 \\ Hospital Manuel Gea González, Ciudad de México; ${ }^{3}$ Servicio de Terapia Intensiva en Ginecología, Hospital General de México Eduardo Liceaga, \\ Ciudad de México; ${ }^{4}$ Servicio de Ginecología y Obstetricia, Hospital General de México Eduardo Liceaga, Ciudad de México. México
}

\section{Resumen}

Antecedentes: La reanimación hídrica intravenosa es esencial en el manejo de los pacientes hospitalizados, en especial en aquellos con enfermedad aguda o crítica. En la actualidad se proponen cuatro premisas con cuatro indicaciones, cuatro preguntas y cuatro fases para la reanimación hídrica intravenosa. Objetivo: El objetivo de esta revisión es dar a conocer esta propuesta de manejo en la reanimación hídrica intravenosa. Conclusiones: Estas premisas de reanimación hídrica intravenosa coexisten de manera continua y con una variabilidad observada en el balance hídrico que se da a entender como un proceso dinámico, sin un patrón fijo temporal ni una escala de tiempo, el cual debe ser individualizado según el contexto clínico del paciente.

PALABRAS CLAVE: Choque. Reanimación hídrica. Balance hídrico. Enfermedad crítica.

\begin{abstract}
Background: Intravenous fluid therapy is essential in the management of hospitalized patients, especially in those with acute or critical illness. It has been proposed four premises, four indications, four questions, and four phases for guidance of this fluid therapy. Objective: The objective of this manuscript is to review these new concepts of intravenous fluid therapy. Conclusion: These phases of intravenous fluid resuscitation coexist continuously and with a variability observed on fluid balance, is meant as a dynamic process, not as a temporary fixed pattern or a time scale and which must be individualized to the clinical context of patient.
\end{abstract}

KEY WORDS: Shock. Reanimation. Fluid balance. Critical illness.

\section{Antecedentes}

La reanimación hídrica intravenosa es una terapia esencial en el manejo de los pacientes hospitalizados, en especial en aquellos con enfermedad aguda o crítica. Mientras que el uso correcto de esta terapia es necesario para asegurar la supervivencia del enfermo en estado de choque, la literatura actual ha demostrado que no está libre de riesgo. Es un hecho que el uso de ciertas soluciones, el volumen infundido y las fallas orgánicas relacionadas con la misma enfermedad ocasionan un incremento en la morbilidad y en la mortalidad.

En el año 2006, el estudio SOAP ${ }^{1}$ demostró que es un factor pronóstico directamente relacionado con la muerte en pacientes con sepsis. Posteriormente, el estudio VASST ${ }^{2}$ demostró la abrumadora disminución

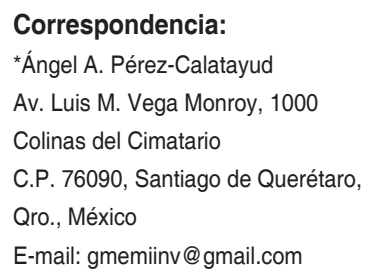

Cir Cir. 2018;86:359-365

Contents available at PubMed www.cirugiaycirujanos.com 
de la supervivencia en los pacientes con sepsis secundaria a la presencia de un balance positivo durante la resucitación y al día 4 , con un incremento de dos veces en el riesgo de muerte.

En la actualidad se ha propuesto un modelo de reanimación hídrica intravenosa en pacientes con sepsis grave que se ha adoptado como estándar en el manejo de estos pacientes en estado crítico.

Por dosis administrada de una solución, la toxicidad depende del tipo y de la composición del fluido administrado, de la fisiología de la enfermedad y de la susceptibilidad del paciente. El debate con respecto al riesgo relativo y al beneficio del uso de coloides y cristaloides ha persistido por años, y en la actualidad la decisión de reanimar con una solución determinada se toma en mayor proporción por preferencia individual y no por la evidencia existente en estudios clínicos de calidad.

Esta revisión resume los conceptos actuales en la reanimación hídrica, haciendo énfasis en la individualización y la reducción de los riesgos para mejorar la evolución del enfermo, mediante el uso de protocolos de actuación por concepto de reanimación.

\section{Marco conceptual para la reanimación hídrica en el paciente grave}

En los últimos años, dentro del manejo de la reanimación hídrica intravenosa del paciente en estado crítico se han propuesto fases de manejo interrelacionadas para la optimización del volumen intravascular y de la hemodinámica, de acuerdo con el estado clínico del paciente ${ }^{3}$.

El grupo de investigación de ADQI XII (Acute Dialysis Quality Iniciative XII) propone un marco conceptual de manejo basado en riesgos relacionados con la fluidoterapia, debido a que un $20 \%$ de los pacientes la reciben de manera inapropiada. El modelo propone tratar la fluidoterapia como cualquier fármaco, considerando la farmacocinética, la farmacodinámica y la toxicidad, con la finalidad de reducir el riesgo de complicaciones ${ }^{4}$.

En un metaanálisis realizado por Malbrain, et al. ${ }^{5}$ se demuestra que una terapia restrictiva, independientemente del tipo de solución manejada, tiene un efecto benéfico sobre la mortalidad, los días de estancia en terapia intensiva y la presencia de hipertensión abdominal.

Este modelo epidemiológico de balance hídrico en el paciente en estado crítico con lesión renal aguda puede extenderse por todo el espectro de la enfermedad crítica ${ }^{6,7}$.
En la actualidad se proponen cuatro premisas con cuatro iniciativas cada una.

\section{Primera premisa: las $4 D$}

Se compone del uso de las $4 \mathrm{D}$ de los antibióticos, y así vamos a iniciar la fluidoterapia considerando:

1. Droga: el tipo de solución a infundir. En este rubro existe todavía un gran debate sobre cuál sería la mejor solución según el escenario del paciente. En la tabla 1 se resumen algunas de las conclusiones actuales de acuerdo a la evidencia existente.

2. Dosis: qué cantidad de solución necesitamos infundir. Esto dependerá del escenario clínico del paciente.

3. Duración: cuánto tiempo se va a infundir.

4. Desescalamiento: cuándo suspender el volumen infundido.

Estas premisas se relacionan directamente con la propuesta de las fases de la reanimación ${ }^{5}$ (cuarta premisa).

\section{Segunda premisa: las cuatro indicaciones de la terapia hídrica intravenosa}

En la actualidad se conoce que la terapia hídrica intravenosa es esencial en el manejo del paciente hospitalizado, principalmente con cuatro indicaciones: reanimación, mantenimiento, reemplazo y nutrición.

\section{Tercera premisa: las cuatro preguntas de la terapia hídrica}

Una vez que se decide iniciar la terapia hídrica, debemos hacernos siempre las siguientes cuatro preguntas:

1. ¿Cuándo iniciar la terapia hídrica?

Existen numerosos disparadores que hacen que un médico inicie la administración de líquidos intravenosos. De manera tradicional, la manera de valorar el volumen intravascular se realiza por parámetros macrocirculatorios, como son la presencia de hipotensión, taquicardia, etc., o por parámetros asociados a la perfusión por la circulación periférica, como el tiempo de llenado capilar, el gradiente de temperatura central-tobillo o el moteado de la pie ${ }^{9-11}$. Así mismo, los clínicos observan la alteración de la perfusión en relación con la función de órganos, como la presencia de disfunción cognitiva aguda, oliguria y taquipnea. 
Tabla 1. Consideraciones actuales en el uso de soluciones intravenosas

\footnotetext{
- Las soluciones isotónicas o balanceadas deben usarse para la resucitación de primera línea.

- Considerar solución salina isotónica en caso de hipovolemia o en pacientes con alcalosis.

- Usar hemocomponentes en pacientes con hemorragia activa.

- Considerar coloides en pacientes con hipovolemia grave como segunda línea.

- Usar cristaloides en la estabilización prehospitalaria.

- Usar hemocomponentes en pacientes con hemorragia en el perioperatorio y en pacientes con traumatismos.

- Todas las soluciones pueden causar edema intersticial.

- La albúmina ha mostrado beneficio en el manejo de pacientes con sepsis y choque séptico.

- La albúmina está contraindicada en pacientes con traumatismo craneoencefálico.

- Considerar la seguridad de otros coloides en el traumatismo craneoencefálico.

- La soluciones tampón se recomienda en pacientes de cirugía mayor y en pacientes quemados.

- La solución salina es la solución de elección en pacientes con traumatismo craneoencefálico.

- La seguridad de los coloides sintéticos no ha sido demostrada en ninguna población de pacientes.

- Los almidones están contraindicados en pacientes con sepsis.

- Los almidones están contraindicados en pacientes con riesgo de insuficiencia renal aguda.

- La seguridad de las soluciones salinas hipertónicas no ha sido establecida en ninguna población de pacientes.

- No se debe usar solución salina en pacientes con acidosis metabólica (riesgo de acidosis hiperclorémica).
}

Sin embargo, todos estos parámetros clínicos carecen de especificidad y pueden tener causas alternativas, no relacionadas con la hipoperfusión por hipovolemia. Los biomarcadores como el lactato, marcador del metabolismo anaerobio, han sido manejados como meta en reanimación, pero también han sido causa de controversia, pues no siempre se ve el efecto de la estimulación de la bomba Na-K-ATPasa por la epinefrina en estados hiperadrenérgicos como la sepsis, el traumatismo o diferentes estados de choque. La hemoconcentración manifestada por el hematocrito o por la concentración de proteínas también se ha considerado una indicación de iniciar la reanimación hídrica intravenosa en pacientes de áreas críticas. De los parámetros estáticos, en la actualidad solo la saturación venosa central se utiliza en las guías de práctica clínica para la reanimación. Por último, los parámetros dinámicos, los cuales se recomiendan para guiar la optimización hemodinámica del paciente grave, se basan principalmente en la idea de la función cardiaca y en la curva de Starling como premisa de la hipoperfusión ${ }^{8}$.

2. ¿Cuándo detener la terapia hídrica intravenosa? A pesar de que la sobrecarga hídrica se asocia a una mala evolución, en la literatura actual no existen marcadores específicos para detener la administración de líquidos. La recomendación de los expertos se enfoca solo a evitar la sobrecarga hídrica. Sin embargo, uno no debe detener la administración de líquidos antes ${ }^{12}$. En la literatura se encuentran diferentes variables clínicas y experimentales. Signos de sobrecarga hídrica, edema periférico y pulmonar son marcadores claros de esta situación, y además pueden indicar una mala distribución de los líquidos en ausencia de sobrecarga hídrica franca, pero estos signos, junto con el incremento de peso, son marcadores tardíos y no pueden ser usados en el perioperatorio ni en unidades de terapia intensiva. Dentro de los exámenes de laboratorio, la depuración del lactato para guiar la terapia hídrica intravenosa en estados de choque, así como la normalización de este parámetro, podrían usarse como marcadores para detener la terapia hídrica intravenosa ${ }^{13}$. Dentro de los parámetros estáticos, el incremento de las presiones de llenado, como es la presión en cuña de la arteria pulmonar o la presión venosa central (no para respuesta a volumen, pero sí para darnos una idea de la tolerancia a la administración de volumen), podrían marcar un límite a la reanimación. Por último, los parámetros dinámicos ${ }^{14}$, en general la ausencia 
de un incremento significativo posterior a la prueba de elevación de las piernas o a un reto de líquidos, son indicadores sensibles y específicos para no continuar la administración de líquidos. El agua pulmonar extravascular ha sido evaluada para la cuantificación del edema pulmonar agudo y de la formación de edema ${ }^{15,16}$.

3. ¿Cuándo iniciar la desresucitación?

A pesar de la evidencia, no existen en la actualidad marcadores clínicos ni de laboratorio para iniciar la desresucitación en los pacientes críticos. Sin embargo, por consenso de los expertos se establecen las siguientes recomendaciones. Se deben manejar protocolos de intervención para evitar la sobrecarga hídrica, lo que significa el uso de parámetros dinámicos, vasopresores tempranos en estado de choque y evaluación de la respuesta a volumen. Esto es de vital importancia en los pacientes con sepsis, hipertensión intraabdominal y síndrome de insuficiencia respiratoria aguda. Se recomienda que después de la fase de reanimación (cuarta premisa) el balance sea neutro o negativo para los días 3 al 7; esto incluye el uso de diuréticos en los pacientes con función renal conservada o el uso de ultrafiltración por medio de tratamiento sustitutivo renal (baja evidencia) ${ }^{8}$. A la pregunta de por qué iniciar la desresucitación solo se puede contestar que la sobrecarga hídrica es causa de un incremento de la morbilidad y de la mortalidad en los pacientes graves ${ }^{8}$.

4. ¿Cuándo detener la desresucitación?

Sobre esta cuestión no se tiene suficiente evidencia como para hacer una recomendación, pero debe suponerse que una desresucitación activa podría ocasionar hipoperfusión y más daño al paciente. Se debe mantener un balance neutro o ligeramente negativo, y utilizar las herramientas de monitoreo que se tengan a la mano ${ }^{8}$.

\section{Cuarta premisa: las cuatro fases de la reanimación hídrica intravenosa}

Las cuatro fases para la reanimación hídrica intravenosa de los pacientes en estado de choque son: una fase inicial o de rescate cuya prioridad es realizar las medidas para restaurar la perfusión tisular y el volumen intravascular; una fase de optimización para mantener el volumen intravascular restaurado; una fase de

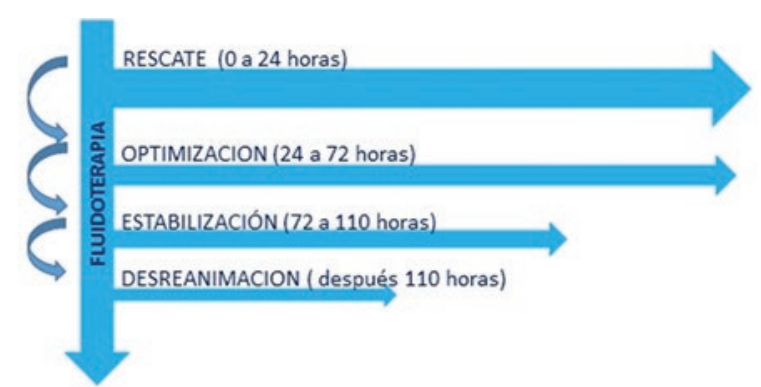

Figura 1. Comportamiento del volumen administrado en las diferentes fases de la resucitación.

estabilización para prevenir el daño de órganos diana tras la estabilización hemodinámica; y una fase de desresucitación en la que se retira el soporte y se restaura la función hemodinámica intrínseca (Fig. 1).

\section{FASE DE RESCATE ${ }^{4}$}

Durante esta fase, entre las horas 0 y 24 , cuando existe hipovolemia sintomática, se administra la mayor parte de las soluciones. Esta fase anticipa y escala de manera inmediata la terapia hídrica para la resucitación del paciente con choque grave (caracterizado por hipotensión, hipoperfusión o ambas), y se caracteriza por el uso de reto de líquidos o bolos de solución intravenosa ${ }^{4}$ (tabla 2).

Durante esta fase se prefiere el uso de cristaloides como primera línea de manejo, con excepción de los hemocomponentes cuando se ameriten. La albúmina podría tener lugar en esta fase para la resucitación en los pacientes con sepsis $6,17,18$, está contraindicado su uso en caso de traumatismo craneoencefálico ${ }^{19}$.

Se recomienda un reto de líquidos a una dosis de 20-30 ml/kg, principalmente como tratamiento de la hipovolemia. La evidencia que apoya el uso de esta terapia es limitada y se basa principalmente en recomendaciones de consenso y guías de práctica clíni$\mathrm{ca}^{20}$. Actualmente existen datos emergentes acerca de que dosis menores obtienen el efecto hemodinámico deseado ${ }^{21}$.

La efectividad y la seguridad de la resucitación con bolo de cristaloides es controversial. El estudio FEAST $^{22}$, realizado en población pediátrica y en un centro de recursos limitados, reportó un incremento de la mortalidad en el grupo de resucitación por bolos a las 48 horas (Riesgo Relativo (RR): 1.45; Intervalo de Confianza (IC) 95\%: 1.13-1.86; $p=0.003$ ), y los autores concluyeron que la causa principal fue el colapso cardiovascular y no la sobrecarga de volumen o factores neurológicos, lo que sugiere que puede 
Tabla 2. Características de las diferentes fases de la reanimación hídrica intravenosa

\begin{tabular}{|c|c|c|c|c|}
\hline Característica & Rescate & Optimización & Estabilización & Desresucitación \\
\hline Metas principales & $\begin{array}{l}\text { Soporte vital } \\
\text { Corregir estado de choque }\end{array}$ & $\begin{array}{l}\text { Rescate orgánico } \\
\text { Optimizar } \\
\text { y rescatar perfusión tisular }\end{array}$ & $\begin{array}{l}\text { Rescate orgánico } \\
\text { Lograr balance hídrico } \\
\text { negativo o neutro }\end{array}$ & $\begin{array}{l}\text { Recuperación orgánica } \\
\text { Movilizar líquido acumulado }\end{array}$ \\
\hline Tiempo (usual) & Minutos & Horas & Días & Días a semanas \\
\hline Fenotipo & Choque grave & Inestable & Estable & Recuperación \\
\hline Reanimación hídrica & Bolos o cargas rápidas & $\begin{array}{l}\text { Infusión hídrica } \\
\text { conservadora y uso de reto } \\
\text { de líquidos }\end{array}$ & $\begin{array}{l}\text { Infusión mínima para } \\
\text { mantener el balance } \\
\text { adecuado con } \\
\text { pérdidas }\end{array}$ & $\begin{array}{l}\text { Movilización de líquido a } \\
\text { balance negativo e iniciar vía } \\
\text { oral de ser posible }\end{array}$ \\
\hline Escenario clínico & $\begin{array}{l}\text { Choque séptico } \\
\text { Traumatismo mayor }\end{array}$ & $\begin{array}{l}\text { Quemaduras, cetoacidosis } \\
\text { diabética } \\
\text { Terapia dirigida a metas en } \\
\text { el intraoperatorio }\end{array}$ & $\begin{array}{l}\text { Manejo en pancreatitis } \\
\text { aguda }\end{array}$ & $\begin{array}{l}\text { Fase de recuperación de } \\
\text { enfermedad aguda, en nutrición } \\
\text { enteral completa } \\
\text { Recuperación de ataque renal }\end{array}$ \\
\hline
\end{tabular}

existir una interacción adversa entre la administración de soluciones intravenosas y la respuesta autonómica compensatoria.

El uso temprano de vasopresores, como la norepinefrina, se recomienda como terapia adjunta durante la resucitación para reducir el volumen necesario a infundir y mejorar la perfusión tisular por incremento del retorno venoso, la presión arterial media y el gasto cardiaco.

\section{FASE DE OPTIMIZACIÓN 5,8}

En esta fase, entre las horas 24 y 72 , el índice de hipovolemia se ha reducido de manera significativa, por lo que se necesitan volúmenes menores para la resucitación (5 y $15 \mathrm{ml} / \mathrm{kg}$ ). Durante esta fase el paciente no tiene peligro inminente de muerte; se encuentra en una fase compensada del estado de choque (con riesgo de descompensación) y la administración de soluciones debe realizarse de manera juiciosa, con la finalidad de optimizar el gasto cardiaco, mejorar la perfusión tisular y, como objetivo principal, mitigar la disfunción orgánica ${ }^{5}$. En esta fase se utiliza el reto de líquidos para observar la respuesta hemodinámica del paciente a la administración de volumen intravascular y evitar así la sobrecarga hídrica.

No existe evidencia científica suficiente que muestre que esta maniobra terapéutica mejore la presión arterial media, la perfusión sistémica o la recuperación de la falla orgánica, como se observa en enfermos con insuficiencia renal aguda ${ }^{23}$. La mayoría de los fluidos administrados, en especial los cristaloides, se acumulan en el tejido intersticial en condiciones de aumento de la permeabilidad tisular por fuga capilar. El efecto neto de la administración de volumen innecesario e inefectivo no optimiza la función hemodinámica sistémica, y aumenta el balance acumulado con la consecuente formación de edema intersticial iatrogénico y un incremento de la mortalidad, de los días de ventilación mecánica y de la necesidad de terapia de reemplazo renal, en especial en los pacientes con sepsis ${ }^{11}$ y síndrome de insuficiencia respiratoria aguda ${ }^{24}$.

\section{FASE DE ESTABILIZACIÓN ${ }^{5}$}

Esta fase ocurre durante las 72 a las 96 horas y refleja el punto en que el paciente se encuentra estable. Se distingue de las primeras dos fases por la ausencia de choque (compensado o descompensado), y la terapia hídrica de esta fase solo es de mantenimiento. En esta fase debe lograrse un balance hídrico acumulado neutro o negativo (Fig. 1).

Existe poca evidencia en la actualidad sobre el efecto de las estrategias de reanimación restrictiva minimizando el balance hídrico acumulado ${ }^{25}$.

\section{FASE DE DESRESUCITACIÓN $N^{5,8}$}

La fase de desresucitación o desescalamiento, que ocurre comúnmente a las 96 horas de manejo o cuando se logra la estabilidad hemodinámica, es para lograr un balance hídrico negativo por restricción en la administración de líquidos intravenosos o mediante la remoción de fluidos por inducción de la diuresis espontánea o con diuréticos.

Una estrategia para eliminar el exceso de fluidos acumulados en las fases previas parece ser más 
intuitiva; sin embargo, una estrategia de restringir el uso excesivo e innecesario podría ser efectiva ${ }^{26}$. No hay evidencia suficiente acerca del uso de hemofiltración para lograr esta meta en ausencia de insuficiencia renal aguda ${ }^{23}$.

Los efectos adversos del edema intersticial pueden no ser prevenidos por la simple remoción del exceso de fluidos, especialmente en la administración de soluciones con toxicidad dependiente de la dosis, como los almidones. La estrategia de un uso restrictivo de líquidos se asocia a un mal pronóstico neuropsicológico en los pacientes con síndrome de insuficiencia respiratoria aguda ${ }^{27}$.

\section{Toxicidad hídrica}

La acumulación hídrica está asociada a un incremento en la morbimortalidad, y en especial al desarrollo de insuficiencia renal aguda ${ }^{28}$.

En los pacientes con insuficiencia renal aguda asociada a sepsis, el uso de soluciones intravenosas a un volumen continuo no incrementa la recuperación de la función renal y se asocia a un deterioro de la función pulmonar con alteraciones en la oxigenación ${ }^{29}$. Estudios observacionales en pacientes críticos muestran que la presencia de insuficiencia renal aguda asociada a sepsis es un predictor de mortalidad a 60 días (RR: 1.21/l por 24 h; IC 95\%: 1.13-1.28; $p<0.001)^{30}$. Adicionalmente, a pesar de que el estudio FACCT no demostró diferencias en la mortalidad entre la reanimación hídrica conservadora y la reanimación hídrica liberal en pacientes con lesión pulmonar aguda, la terapia conservadora mostró una tendencia a una disminución de los días de estancia hospitalaria, una menor necesidad de terapia de reemplazo renal y una mejor función pulmonar ${ }^{25}$.

El incremento en la acumulación de fluidos en pacientes con insuficiencia renal aguda, adultos y pediátricos, al momento de iniciar una terapia de reemplazo renal, se asocia a un incremento de la mortalidad y disminuye la probabilidad de recuperación de la función renal. Por cada $1 \%$ de incremento en la sobrecarga de volumen al inicio de la terapia de reemplazo renal existe un incremento en el riesgo de muerte del $3 \%{ }^{31-35}$.

El fracaso en la apreciación de las fases de la reanimación hídrica puede subestimar el fenómeno denominado fluid creep, identificado por primera vez en pacientes quemados, en respuesta al entusiasmo por la resucitación agresiva y mantenida ${ }^{36}$. Estas observaciones remarcan la importancia del monitoreo del balance hídrico en el paciente crítico, en particular tras la fase de rescate, cuando los ingresos (p. ej., medicamentos, productos sanguíneos, etc.) pueden exceder los egresos (p. ej., oliguria, ataque renal) y llevar a una sobrecarga hídrica ${ }^{37}$.

\section{Conclusiones}

Numerosos estudios en enfermos críticos y quirúrgicos han demostrado la mala evolución de los pacientes con sobrecarga hídrica y el impacto de esta en la morbilidad y la mortalidad. Las fases de la reanimación hídrica intravenosa coexisten de manera continua. La variabilidad observada en el balance hídrico se entiende como un proceso dinámico, sin un patrón fijo temporal ni una escala de tiempo, y debe ser individualizado según el contexto clínico del paciente. El manejo inapropiado de la fluidoterapia, independientemente del tipo de solución o fluido que se utilice, puede lesionar los mecanismos compensatorios. Aunque estos conceptos son utilizados actualmente como protocolo de actuación en la resucitación hídrica del paciente crítico, aún se necesitan estudios que los validen tanto en su aplicación clínica como en investigación básica ${ }^{25}$.

\section{Conflicto de intereses}

Todos los autores declaran no tener conflictos de intereses.

\section{Bibliografía}

1. Vincent JL, Sakr Y, Sprung CL, Ranieri VM, Reinhart K, Gerlach H, et al. Sepsis in European intensive care units: results of the SOAP study. Sepsis Occurrence in Acutely III Patients Investigators. Crit Care Med. 2006;34:344-53.

2. Boyd JH, Forbes J, Nakada T, Walley KR, Russell JA. Fluid resuscitation in septic shock: a positive fluid balance and elevated central venous pressure are associated with increased mortality. Crit Care Med. 2011;39:259.

3. Goldstein SL. Fluid management in acute kidney injury. J Intensive Care Med. 2014;29:183-9.

4. Hoste EA, Maitland K, Brudney CS, Mehta R, Vincent JL, Yates D, et al. ADQI XII Investigators Group. Four phases of intravenous fluid therapy: a conceptual model. Br J Anaesth. 2014;113:740-7.

5. Malbrain MLNG, Marik PE, Witters I, Cordemans C, Kirkpatrick AW, Roberts DJ, et al. Fluid overload, de-resuscitation, and outcomes in critically ill or injured patients: a systematic review with suggestions for clinical practice. Anaesthesiol Intensive Ther. 2014;46:361-80.

6. Finfer S, Liu B, Taylor C, Bellomo R, Billot L, Cook D, et al.Resuscitation fluid use in critically ill adults: an international cross-sectional study in 391 intensive care units. Crit Care. 2010;14:R18.

7. McDermid RC, Raghunathan K, Romanovsky A, Shaw AD, Bagshaw SM. Controversies in fluid therapy: type, dose and toxicity. World J Crit Care Med. 2014;3:24-33.

8. Veenstra G, Ince C, Boerma EC. Direct markers of organ perfusion to guide fluid therapy: when to start, when to stop. Best Pract Res Clin Anaesthesiol. 2014;28:217-26.

9. Lima A, Jansen TC, van Bommel J, Ince C, Bakker J. The prognostic value of the subjective assessment of peripheral perfusion in critically ill patients. Crit Care Med. 2009;37:934-8. 
10. Joly HR, Weil MH. Temperature of the great toe as an indication of the severity of shock. Circulation. 1969;39:131e8.

11. Ait-Oufella H, Lemoinne S, Boelle PY, Galbois A, Baudel JL, Lemant J, et al. Mottling score predicts survival in septic shock. Intensive Care Med. 2011;37(5): 801-7.

12. Ricci Z, Ronco C. Year in review 2012: critical care e nephrology. Crit Care. 2013;17:246.

13. Jansen TC, van Bommel J, Schoonderbeek FJ, Sleeswijk Visser SJ, van der Klooster JM, Lima AP, et al. Early lactate-guided therapy in intensive care unit patients: a multicenter, open-label, randomized controlled trial. Am J Respir Crit Care Med. 2010;182:752-61.

14. Trof RJ, Danad I, Reilingh MWL, Breukers RM, Groeneveld AB. Cardiac filling volumes versus pressures for predicting fluid responsiveness after cardiovascular surgery: the role of systolic cardiac function. Crit Care. 2011;15:R73

15. Jansen JRC, Maas JJ, Pinsky MR. Bedside assessment of mean systemic filling pressure. Curr Opin Crit Care. 2010;16:231-6.

16. Michard F. Bedside assessment of extravascular lung water by dilution methods: temptations and pitfalls. Crit Care Med. 2007;35:1186-92.

17. Finfer S, McEvoy S, Bellomo R, McArthur C, Myburgh J, Norton R. Impact of albumin compared to saline on organ function and mortality of patients with severe sepsis. Intensive Care Med. 2011;37:86-96.

18. Caironi P, Tognoni G, Masson S, Fumagalli R, Pesenti A, Romero M, et al. Albumin replacement in patients with severe sepsis or septic shock. N Engl J Med. 2014;370:1412-21.

19. Cooper DJ, Myburgh J, Heritier S, Finfer S, Bellomo R, Billot L, et al. Albumin resuscitation for traumatic brain injury: is intracranial hypertension the cause of increased mortality? J Neurotrauma. 2013;30:512-8.

20. Dellinger RP, Levy MM, Rhodes A, Annane D, Gerlach H, Opal SM, et al. Surviving sepsis campaign: international guidelines for management of severe sepsis and septic shock, 2012. Intensive Care Med. 2013;39: 165-228.

21. Yealy DM, Kellum JA, Huang DT, Barnato AE, Weissfeld LA, et al. ProCESS Investigators. A randomized trial of protocol-based care for early septic shock. N Engl J Med. 2014;370:1683-93.

22. Maitland K, Kiguli S, Opoka RO, Engoru C, Olupot-Olupot P, Akech SO, et al. Mortality after fluid bolus in African children with shock. N Engl $\mathrm{J}$ Med. 2011;364:2483-95

23. Prowle JR, Bellomo R. Fluid administration and the kidney. Curr Opin Crit Care. 2010;16:332-6.

24. Boyd JH, Forbes J, Nakada TA, Walley KR, Russell JA. Fluid resuscitation in septic shock: a positive fluid balance and elevated central venous pressure are associated with increased mortality. Crit Care Med. 2011;39:259-65.
25. Wiedemann HP, Wheeler AP, Bernard GR, Thompson BT, Hayden D, deBoisblanc $B$, et al. Comparison of two fluid-management strategies in acute lung injury. N Engl J Med. 2006;354:2564-75.

26. Myburgh JA. Fluid resuscitation in acute medicine: what is the current situation? J Intern Med. 2015;277:58-68.

27. Mikkelsen ME, Christie JD, Lanken PN, Biester RC, Thompson BT, Bellamy SL, et al. The adult respiratory distress syndrome cognitive outcomes study: long-term neuropsychological function in survivors of acute lung injury. Am J Respir Crit Care Med. 2012;185:1307-15.

28. Finfer S, Bellomo R, Boyce N, French J, Myburgh J, Norton R. A comparison of albumin and saline for fluid resuscitation in the intensive care unit. N Engl J Med. 2004;350:2247-56.

29. Arikan AA, Zappitelli M, Goldstein SL, Naipaul A, Jefferson LS, Loftis LL. Fluid overload is associated with impaired oxygenation and morbidity in critically ill children. Pediatr Crit Care Med. 2012;13:253-8.

30. Bellomo R, Cass A, Cole L, Finfer S, Gallagher M, Lee J, et al. An observational study fluid balance and patient outcomes in the Randomized Evaluation of Normal vs. Augmented Level of Replacement Therapy trial. Crit Care Med. 2012;40:1753-60.

31. Brunkhorst FM, Engel C, Bloos F, Meier-Hellmann A, Ragaller M, Weiler $\mathrm{N}$, et al. Intensive insulin therapy and pentastarch resuscitation in severe sepsis. N Engl J Med. 2008;358:125-39.

32. Raghunathan K, Shaw A. Hydroxyethyl starch or saline in intensive care. N Engl J Med. 2013;368:774-5.

33. U.S. Food and Drug Administration. Silver Spring, 2013 Hydroxyethyl Starch Solutions: FDA Safety Communication - Boxed Warning on Increased Mortality and Severe Renal Injury and Risk of Bleeding. (Consultado el 1 de febrero de 2016.) Disponible en: http://www.fda.gov/ Safety/MedWatch/SafetyInformation/SafetyAlertsforHumanMedicalProducts/ucm358349.htm.

34. Meybohm P, Van Aken H, De Gasperi A, De Hert S, Della Rocca G, Girbes AR, et al. Re-evaluating currently available data and suggestions for planning randomised controlled studies regarding the use of hydroxyethyl-starch in critically ill patients a multidisciplinary statement. Crit Care. 2013;17:R166.

35. Sutherland SM, Zappitelli M, Alexander SR, Chua AN, Brophy PD, Bunchman TE, et al. Fluid overload and mortality in children receiving continuous renal replacement therapy: the prospective pediatric continuous renal replacement therapy registry. Am J Kidney Dis. 2010;55:316-25.

36. Saffle Jl. The phenomenon of "fluid creep" in acute burn resuscitation. J Burn Care Res. 2007;28:382-95.

37. Bagshaw SM, Brophy PD, Cruz D, Ronco C. Fluid balance as a biomarker: impact of fluid overload on outcome in critically ill patients with acute kidney injury. Crit Care. 2008;12:169. 